\title{
Mosquito transmission of the rodent malaria parasite Plasmodium chabaudi
}

\author{
Philip J Spence*, William Jarra, Prisca Lévy, Wiebke Nahrendorf and Jean Langhorne*
}

\begin{abstract}
Background: Serial blood passage of Plasmodium increases virulence, whilst mosquito transmission inherently regulates parasite virulence within the mammalian host. It is, therefore, imperative that all aspects of experimental malaria research are studied in the context of the complete Plasmodium life cycle.

Methods: Plasmodium chabaudi chabaudi displays many characteristics associated with human Plasmodium infection of natural mosquito vectors and the mammalian host, and thus provides a unique opportunity to study the pathogenesis of malaria in a single infection setting. An optimized protocol that permits efficient and reproducible vector transmission of $P$. c. chabaudi via Anopheles stephensi was developed.

Results and conclusions: This protocol was utilized for mosquito transmission of genetically distinct $P$. c. chabaudi isolates, highlighting differential parasite virulence within the mosquito vector and the spectrum of host susceptibility to infection initiated via the natural route, mosquito bite. An apposite experimental system in which to delineate the pathogenesis of malaria is described in detail.
\end{abstract}

\section{Background}

Plasmodium chabaudi chabaudi establishes synchronous, chronic and recrudescing blood-stage infections in rodents, and exhibits many characteristics associated with the pathogenesis of human malaria, such as rosetting, sequestration and antigenic variation [1]. Genetically distinct isolates of $P$. c c chabaudi display distinct virulence phenotypes [2], and the development of $P . c$. chabaudi specific transfection technology has recently been reported [3]. Plasmodium chabaudi' chabaudi is therefore an ideal species with which to study the interaction between Plasmodium and the mammalian host in an in vivo setting. Nevertheless, it is established that serial blood passage of Plasmodium increases virulence $[4,5]$, and it has been demonstrated that mosquito transmission of serially blood passaged P. c. chabaudi intrinsically modifies the asexual blood-stage parasite, transforming the course and outcome of infection [Spence PJ, Jarra W, Lévy P, Reid AJ, Chappell L, Brugat T, Sanders M, Berriman M, Langhorne J. personal communication]. Mosquito transmission of Plasmodium therefore directly regulates parasite virulence, and is

\footnotetext{
* Correspondence: pspence@nimr.mrc.ac.uk; jlangho@nimr.mrc.ac.uk Division of Parasitology, MRC National Institute for Medical Research, Mill Hill, London NW7 1AA, UK
}

thus an imperative in experimental malaria research. The required conditions for transmission of P. c. chabaudi via the mosquito vector differ markedly from all other rodent malaria species [6,7]. An optimized protocol for mosquito transmission of $P$. c. chabaudi has been developed to facilitate efficient and reproducible transmission of distinct isolates of $P$. c. chabaudi to distinct strains of laboratory mice. Here, this protocol is presented, and details the requirements of transmission and the expected outcomes of infection. Mosquito transmission of P. c. chabaudi permits analysis of parasite virulence, and vector and host responses to infection, in the context of the complete Plasmodium life cycle. An apposite experimental system in which to study many of the hallmark features of human disease is described.

\section{Methods}

Mice, mosquitoes and parasites

Inbred $\mathrm{BALB} / \mathrm{c}, \mathrm{C} 57 \mathrm{BL} / 6$ and $\mathrm{C} 3 \mathrm{H} / \mathrm{HeN}$ mice, and outbred Parkes mice, are bred under specific pathogen-free conditions at the MRC National Institute for Medical Research (NIMR). Experiments are performed in accordance with UK Home Office regulations (PPL 80/2358) and approved by the ethical review panel at the NIMR. Female C57BL/6 mice are routinely used to initiate 
mosquito transmission of $P$. c. chabaudi between six to eight weeks of age and at approximately 20 g body weight. Mice are housed under normal light conditions (light 07.00-19.00 : dark 19.00-07.00) at $22^{\circ} \mathrm{C}, 50 \%$ relative humidity and with continuous access to mouse breeder diet and water.

Anopheles stephensi, strain SD500, are reared at $28^{\circ} \mathrm{C}$, $60 \%$ relative humidity and under normal light conditions (light 07.00-19.00 : dark 19.00-07.00). Egg lays are induced through live rat feeds and larvae are reared at a density of approximately 500 per litre of $\mathrm{dH}_{2} \mathrm{O}$. Larvae are provided with Liquifry No. 1 and pool pellets, whilst adult mosquitoes are provided with Fructose. Female An. stephensi are routinely infected with $P$. c. chabaudi at seven to nine days of age. Following infection, mosquitoes are housed under normal light conditions at $26^{\circ}$ C, $60 \%$ relative humidity and with continuous access to Fructose + PABA.

Frozen stocks of serially blood passaged $P$. c. chabaudi (isolates $\mathrm{AS}$ and $\mathrm{CB}$ ) are maintained in liquid nitrogen, and can be obtained from the European Malaria Reagent Repository, University of Edinburgh. Plasmodium chabaudi chabaudi is routinely transmitted between C57BL/6 mice and An. stephensi. To initiate transmission, frozen parasite stocks are supplemented with a 0.5 volume of Krebs saline + glucose [3], rapidly thawed by hand and injected intraperitoneally (ip) into a single C57BL/6 mouse. Peripheral parasitaemia is monitored daily on thin blood smears and parasitized erythrocytes (pE) are isolated at a parasitaemia of 5-10\%, typically day 5 post-injection, and used to infect mice.

\section{Materials}

4-Aminobenzoic acid (PABA; Sigma, cat. no. A9878)

Atipamezole hydrochloride, $5 \mathrm{mg} / \mathrm{ml}$ solution for injection (Atipam; veterinary prescription only)

Autoclavable mouse breeder diet, WO/HS (LabDiet, cat. no. 5021)

Cell culture dish, $60 \times 15 \mathrm{~mm}$ (Corning, cat. no. 430166)

Chromatography filter paper, 3MM (Fisher Scientific, cat. no. FB59515)

Collapsible cage, $30 \mathrm{~cm}^{3}$ (BioQuip Products, Inc., cat. no. $1450 \mathrm{~B})$

Compressed gas: $\mathrm{CO}_{2}$.

Cotton wool balls

Cover slips, glass, $18 \mathrm{~mm}^{2}$ (Menzel Gläser, cat. no. MNJ-350-010 K)

D-(-)-Fructose (Sigma, cat. no. F0127)

Erlenmeyer conical flask, glass, $100 \mathrm{ml}$ (Fisher Scientific, cat. no. FB33131)

FBS Gold (PAA Laboratories, cat. no. A15-649)

Filter paper circles, grade 1, 125mm (Whatman, cat. no. 1001 125)
Gentamicin solution, 50mg/ml (Sigma, cat. no. G1397)

Glass basin, flat base, straight side, $300 \mathrm{ml}$ (VWR, cat. no. 216-2764)

Glucose (BDH (VWR), cat no. 101174Y)

Homogeniser mortar and pestle, $0.1 \mathrm{ml}$ (Fisher Scientific, cat. no. FB56673)

Ketamine hydrochloride, $100 \mathrm{mg} / \mathrm{ml}$ solution for injection (Vetalar V; veterinary prescription only)

Liquifry No. 1 (Interpet)

Medetomidine hydrochloride, $1 \mathrm{mg} / \mathrm{ml}$ solution for injection (Medetor; veterinary prescription only)

Microscope slides, ground edges $45^{\circ}$ (VWR, cat. no. 631-1560)

Micro test tubes, $1.5 \mathrm{ml}$ (Eppendorf, cat. no. 0030 120.086)

Needle, 27G and 30G, 0.5 inch (BD, cat. no. 300635 and 304000)

PBS, pH 7.2 (Invitrogen, cat. no. 20012)

Polypropylene centrifuge tubes, $50 \mathrm{ml}$ (Corning, cat. no. 430291)

Pool pellets (Dr Clarke's pond foods)

RPMI 1640 + L-Glutamine (Invitrogen, cat. no. 21875)

Rubber elastic bands

Sodium bicarbonate (Sigma, cat. no. S6297)

Sodium chloride solution, $0.9 \%$ (saline; Sigma, cat. no. S8776)

Syringe, $1.0 \mathrm{ml}$ (BD, cat. no. 300013)

Tulle fabric netting, white, $12 \mathrm{~cm}^{2}$

Vacuum filter system, PES, $0.22 \mu \mathrm{m}$ pore size, $500 \mathrm{ml}$ (Corning, cat. no. 431097)

White premium paper cups, $12 \mathrm{cl}$ and $25 \mathrm{cl}$ (Benders, cat. no. 820400E and 8209006)

\section{Reagent set-up}

Fructose: $8 \%(\mathrm{w} / \mathrm{v}) \mathrm{D}-(-)$-Fructose in $\mathrm{dH}_{2} \mathrm{O}$. Sterilise by membrane filtration. Store at $4^{\circ} \mathrm{C}$ for up to one month.

Fructose + PABA (F/PABA): 8\% (w/v) D-(-)-Fructose and $0.05 \%(\mathrm{w} / \mathrm{v})$ PABA in $\mathrm{dH}_{2} \mathrm{O}$. Sterilise by membrane filtration. Store at $4^{\circ} \mathrm{C}$ for up to one month and protect from light.

F/PABA + Gentamicin: $50 \mu \mathrm{g} / \mathrm{ml}$ Gentamicin in $\mathrm{F} /$ PABA. Prepare a fresh solution before use.

RPMI + Glucose: $0.2 \%(\mathrm{w} / \mathrm{v})$ sodium bicarbonate, $10 \%$ $(\mathrm{v} / \mathrm{v})$ FCS and $0.2 \%(\mathrm{w} / \mathrm{v})$ glucose in RPMI $1640+\mathrm{L}$ Glutamine. Prepare a fresh solution before use.

Ketamine + Medetomidine: $10 \mathrm{mg} / \mathrm{ml}$ Ketamine hydrochloride and $100 \mu \mathrm{g} / \mathrm{ml}$ Medetomidine hydrochloride in saline. Inject a $20 \mathrm{~g}$ mouse with a $100 \mu \mathrm{l}$ volume to achieve the required working dose of $50 \mathrm{mg} / \mathrm{kg}$ and $500 \mu \mathrm{g} / \mathrm{kg}$, respectively. Prepare a fresh solution before use.

Atipamezole: $1 \mathrm{mg} / \mathrm{ml}$ Atipamezole hydrochloride in saline. Inject a $20 \mathrm{~g}$ mouse with a $50 \mu \mathrm{l}$ volume to achieve 
the required working dose of $2.5 \mathrm{mg} / \mathrm{kg}$. Prepare a fresh solution before use.

\section{Equipment}

Improved Neubauer cell counting chamber, depth $0.1 \mathrm{~mm}$ (Hawksley, cat. no. AC1000)

Insect aspirator, $1.5 \mathrm{~V}$ powered (Hausherr's machine works)

Light microscope, phase contrast, 10 - 40x objectives (Zeiss Axio Scope A1 or equivalent)

Microcentrifuge (Heraeus Fresco 17 or equivalent)

Stereomicroscope, 7.5 - 60x zoom (Leica M80 or equivalent)

Ultrasonic humidity cabinet (LEEC SFC3C/RH or equivalent)

\section{Procedure}

Day -14 | C57BL/6 mice are injected ip with $10^{5} \mathrm{pE}$ and peripheral parasitaemia is monitored by thin blood smear throughout infection. For every 100 mosquitoes that are to be infected $\geq 1$ mouse with a gametocyte density $\geq 0.1 \%$ of total erythrocytes will be required; the number of mice initially infected is, therefore, dependent upon the frequency that will exceed that threshold. Note: Transmission fails if circulating gametocytes are not observed, and transmission efficiency is substantially reduced if gametocytes are observed at a density $<0.1 \%$ of total erythrocytes. Critical: Some isolates of P. c. chabaudi are highly virulent, causing severe disease and mortality, and necessitating increased numbers and careful monitoring of mice.

Day -5 | Up to 1,500 female An. stephensi (at two to four days of age) are transferred to a $30 \mathrm{~cm}^{3}$ collapsible cage, whose base is lined with three sheets of chromatography filter paper. A feeding station (a $100 \mathrm{ml}$ conical flask with a chromatography filter paper wick and cotton wool bung) is filled with $50 \mathrm{ml}$ of F/PABA + Gentamicin and placed within the cage. The cage is transferred to an ultrasonic humidity cabinet and mosquitoes are hereafter maintained continuously at $26^{\circ} \mathrm{C}, 60 \%$ relative humidity. Note: For every mouse that is to be infected 30 mosquitoes are initially transferred to the cage. Although only 20 mosquitoes are ultimately required per mouse, it is necessary to compensate for the attrition of mosquito numbers that will occur over the following 20 days.

Day -2 The feeding station is replaced to provide 50 $\mathrm{ml}$ of $\mathrm{F} / \mathrm{PABA}$.

Day $\mathbf{- 1}$ | The feeding station is removed and sterile $\mathrm{dH}_{2} \mathrm{O}$ is provided by saturating cotton wool balls, placing them into the caps of $50 \mathrm{ml}$ centrifuge tubes and placing these cotton wool caps onto the top of the cage; one cotton wool cap is provided for every 100 mosquitoes. Note: It is important to starve mosquitoes 24 hours prior to transmission.
Day $\mathbf{0}$ | A thin blood smear is prepared from all mice at approximately 14.00 hours; slides are fixed with methanol and stained with Giemsa's working solution [3]. Gametocytes are enumerated in a count of 5,000 erythrocytes, and all mice that equal or surpass the gametocyte density threshold of $0.1 \%$ of total erythrocytes are selected for transmission. Selected mice are anaesthetized by ip injection of Ketamine + Medetomidine and placed ventral side down onto the top of the cage; mosquitoes are allowed to feed for 30 minutes at room temperature. Following transmission, sterile $\mathrm{dH}_{2} \mathrm{O}$ is provided to mosquitoes in cotton wool caps placed on the top of the cage, which is then returned to the ultrasonic humidity cabinet. Mice are culled by an approved method. NOTE: Transmission is performed at 18.00 hours and under low light conditions. CRITICAL: The required temperature for $P$. c. chabaudi fertilization and development within the mosquito vector is $26.0^{\circ} \mathrm{C}(+/-$ $\left.0.5^{\circ} \mathrm{C}\right)$.

Day 1 F/PABA is provided to mosquitoes in cotton wool caps placed on the top of the cage.

Day 2 The cotton wool caps are replaced to provide fresh F/PABA.

Day 4 | The cotton wool caps are removed and a feeding station containing $50 \mathrm{ml}$ of F/PABA is placed within the cage. An egg bowl is similarly placed within the cage, allowing mosquitoes to safely deposit their eggs. To prepare the egg bowl, a $300 \mathrm{ml}$ glass basin is filled to the rim with $\mathrm{dH}_{2} \mathrm{O}$, and a $125 \mathrm{~mm}$ filter paper circle (with a $15 \mathrm{~mm}$ hole cut from the centre) is positioned such that the outer edge of the circle adheres to the rim of the basin and the inner edge of the circle lies under the surface of the water.

Day 6 | The egg bowl is removed, and the feeding station is replaced to provide a further $50 \mathrm{ml}$ of F/PABA.

Day 8 | To visualize oocyst development: 20 mosquitoes are harvested from the cage using an insect aspirator, anaesthetized with $\mathrm{CO}_{2}$ and transferred to a cell culture dish on ice; mosquito midguts are dissected under a stereomicroscope, transferred into PBS on a microscope slide and covered with a glass cover slip; midguts are observed under a light microscope, utilizing phase contrast to visualize and enumerate oocysts. The feeding station is replaced to provide remaining mosquitoes with a further $50 \mathrm{ml}$ of F/PABA. Note: For guidance on the dissection of Plasmodium-infected mosquitoes refer to 'Methods in Anopheles Research' [8].

Day 10 | The feeding station is replaced to provide a further $50 \mathrm{ml}$ of F/PABA.

Day 12 The feeding station is replaced to provide a further $50 \mathrm{ml}$ of F/PABA.

Day 14 | For every mouse that is to be infected, 20 mosquitoes are harvested from the cage using an insect aspirator, anaesthetized with $\mathrm{CO}_{2}$ and transferred to a 
$25 \mathrm{cl}$ paper cup, which is covered with tulle fabric netting and secured with rubber elastic bands. Alternatively, 10 mosquitoes can be transferred to $25 \mathrm{cl}$ paper cups, or single mosquitoes to $12 \mathrm{cl}$ paper cups, to reduce the number of mosquito bites. An additional mosquito cup is prepared for enumerating sporozoites, and $\mathrm{dH}_{2} \mathrm{O}$ is provided to mosquitoes in cotton wool caps placed on the top of the paper cups. Note: It is important to starve the mosquitoes 24 hours prior to transmission. Critical: Mosquitoes are transferred to paper cups 24 hours prior to transmission to provide sufficient recovery time following anaesthesia. Transfer of mosquitoes to paper cups is not required if experimental mice are to be infected via mechanical transmission.

Day 15 | To enumerate sporozoites: 20 mosquitoes are anaesthetized with $\mathrm{CO}_{2}$ and transferred to a cell culture dish on ice; mosquito salivary glands are dissected under a stereomicroscope, transferred into $100 \mu \mathrm{l}$ of RPMI + Glucose in a $0.1 \mathrm{ml}$ homogenizer and maintained on ice; all sets of salivary glands are pooled and gently homogenized for 30 seconds to release sporozoites, and the supernatant is transferred to a $1.5 \mathrm{ml}$ micro test tube; the sample is centrifuged at 3,500 $\mathrm{xg}$ in a microcentrifuge for 3 minutes at $4{ }^{\circ} \mathrm{C}$ and washed $2 \mathrm{x}$ with $500 \mu \mathrm{l}$ RPMI + Glucose; sporozoites are resuspended in $10 \mu \mathrm{l}$ RPMI + Glucose, transferred to a cell counting chamber and enumerated under a light microscope utilizing phase contrast.

For natural transmission of $P$. c. chabaudi via mosquito bite, each mouse is anaesthetized by ip injection of Ketamine + Medetomidine and placed ventral side down onto the top of one mosquito cup; mosquitoes are allowed to feed for 20 minutes at room temperature. Mice are then injected ip with Atipamezole and monitored to recovery. The number of fed mosquitoes in every cup is enumerated immediately by anaesthetizing mosquitoes with $\mathrm{CO}_{2}$, or at a later time by storing the mosquito cups at $-20^{\circ} \mathrm{C}$. Note: Transmission is performed at 18.00 hours and under low light conditions. Critical: Mice must be closely monitored for recovery from anaesthesia; for example, by testing their righting reflex.

For mechanical transmission of $P$. c. chabaudi via injection, mosquito salivary glands are dissected and homogenized (as described above) and sporozoites washed $3 \mathrm{x}$ with $1 \mathrm{ml}$ RPMI + Glucose. Sporozoites are resuspended in $100 \mu \mathrm{l}$ RMI + Glucose, enumerated on a cell counting chamber using $10 \mu \mathrm{l}$ of sample, further diluted in RPMI + Glucose to the required concentration, and injected intravenously (iv) or intradermally (id) into mice using $27 \mathrm{G}$ or $30 \mathrm{G}$ needles, respectively. Note: To obtain sufficient sporozoite numbers, assume that 100 mosquitoes will yield approximately 25,000 (+/10,000) sporozoites. Critical: Dissected salivary glands and isolated sporozoites should be maintained throughout on ice, and the time from dissection to injection should be minimized to maximize sporozoite viability.

\section{Results}

Plasmodium chabaudi chabaudi (isolates AS and CB) is routinely transmitted between wild-type C57BL/6 mice and $A n$. stephensi utilizing this protocol (Figure 1). All available data from mosquito transmission of $P$. c. chabaudi over a one-year period (18 independent experiments) has been collated, and the results described herein derive from that pooled dataset, unless stated otherwise.

Infection of C57BL/6 mice with serially blood passaged P. c. chabaudi AS via ip injection of $\mathrm{pE}$ leads to an early peak of circulating gametocytes, concurrent with the peak of asexual blood-stage parasitaemia, and a late peak of circulating gametocytes of increased magnitude that follows peak parasitaemia (Figure 2a). A similar kinetic of gametocytosis is observed in BALB/c mice, another widely used inbred laboratory strain. Transmission of $P$. c. chabaudi AS to An. stephensi early in infection (days 5,6 or 7) is inefficient, whereas transmission late in infection (days 13 or 14) is highly efficient (data not shown). Importantly, An. stephensi must feed only on mice with a circulating gametocyte density $\geq 0.1 \%$ of total erythrocytes (at a ratio of $\geq 1$ mouse to 100 mosquitoes) to maximize efficiency of transmission; of $526 \mathrm{C} 57 \mathrm{BL} / 6$ mice infected with $P$. c. chabaudi AS, 408 mice (77.6\%) were equal to or surpassed this threshold on day 14 post-infection. Under these conditions, transmission of P. c. chabaudi AS results in infection of $71.5 \%$ (55\% $85 \%)$ (median with range) of mosquitoes; of $311 \mathrm{An}$. stephensi infected with P. c. chabaudi AS, 227 (73\%) harboured between 1-20 oocysts on day 8 post-infection (Figure 2b). On day 14 post-infection, the number of salivary gland sporozoites in An. stephensi infected with $P$. c. chabaudi AS is 437.5 (43.2-956) (median with range) (Figure 2c). Interestingly, P. c. chabaudi CB, an isolate with increased virulence within the mammalian host, displays increased transmissibility, and An. stephensi infected with P. c. chabaudi CB harbour increased oocyst and sporozoite burdens (Figure 2b-c).

Sporozoites obtained from salivary glands of An. stephensi on day 15 of infection with P. c. chabaudi AS are highly infective; iv injection of 10 sporozoites is sufficient to elicit patent blood-stage parasitaemia in $20 \%$ of C57BL/6 mice, whilst 100 sporozoites ensures patent parasitaemia in $100 \%$ of mice (Figure 3a). Inoculation of sporozoites by id injection is comparatively ineffectual; 1,000 sporozoites results in just $60 \%$ of mice with patent parasitaemia. In contrast, transmission of $P$. c. chabaudi AS via mosquito bite is highly efficient; one third of all C57BL/6 mice exposed to single mosquito bites develop 


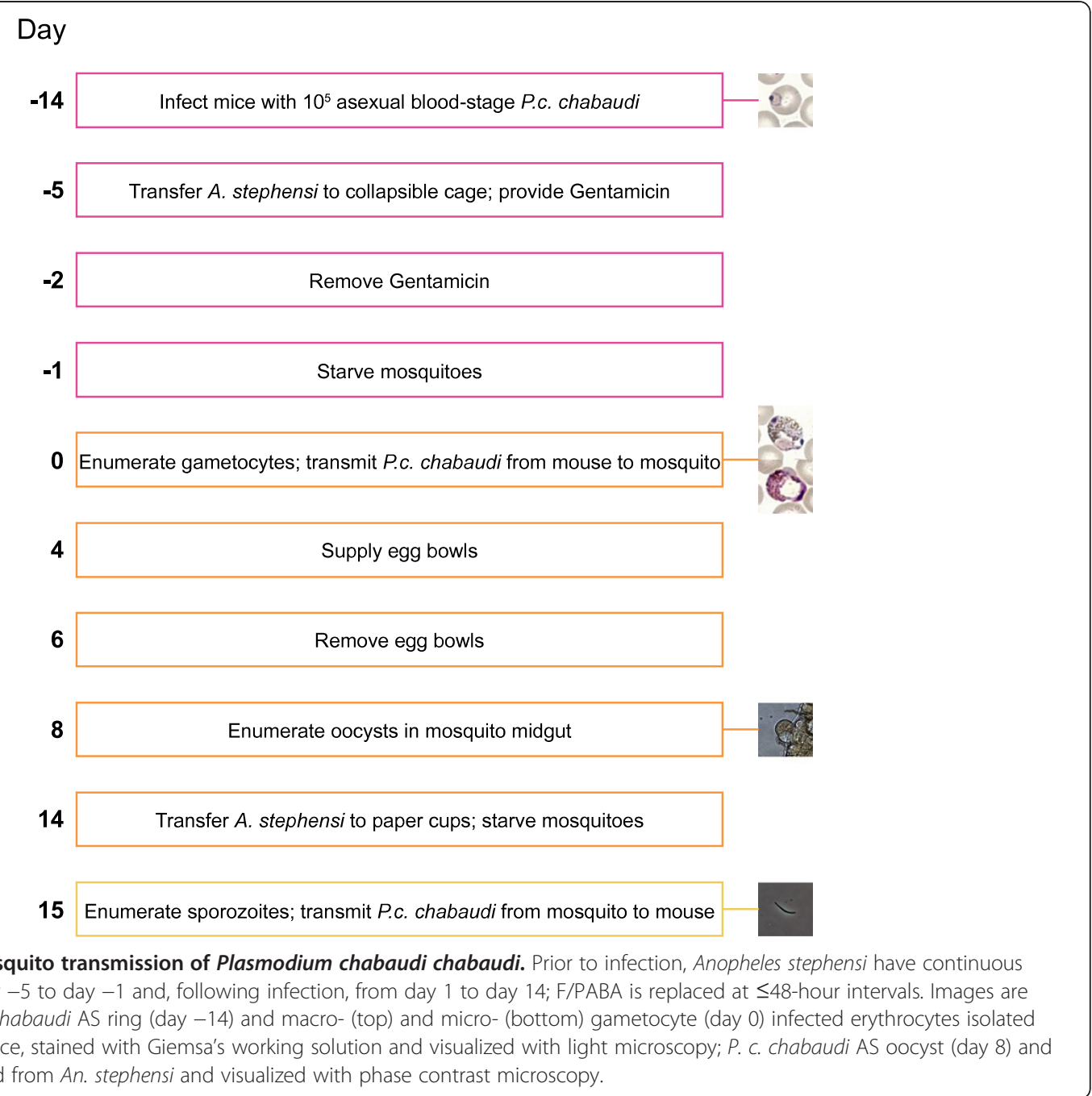

Figure 1 Timeline of mosquito transmission of Plasmodium chabaudi chabaudi. Prior to infection, Anopheles stephensi have continuous access to F/PABA from day -5 to day -1 and, following infection, from day 1 to day 14; F/PABA is replaced at $\leq 48$-hour intervals. Images are shown for reference: P. c. chabaudi AS ring (day -14) and macro- (top) and micro- (bottom) gametocyte (day 0) infected erythrocytes isolated from wild-type C57BL/6 mice, stained with Giemsa's working solution and visualized with light microscopy; P. c. chabaudi AS oocyst (day 8) and sporozoite (day 15) isolated from An. stephensi and visualized with phase contrast microscopy.

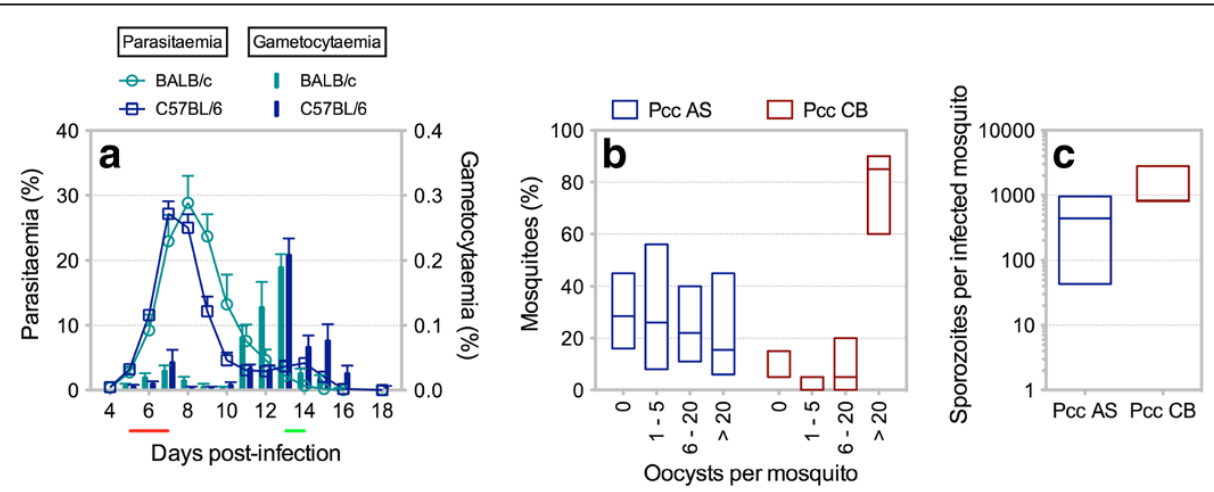

Figure 2 Gametocytosis and sporogony in Plasmodium chabaudi chabaudi. a, Parasitaemia and gametocytaemia of BALB/C and C57BL/6 mice infected with P. c. chabaudi AS by ip injection of $10^{5} \mathrm{pE}(\mathrm{n}=$ six per group; data are presented as mean with SEM). Transmission efficiency to Anopheles stephensi is highlighted under the graph: low efficiency (red line) versus high efficiency (green line). $\mathbf{b}-\mathbf{c}$, Number of oocysts in the midguts of An. stephensi on day 8 (b) and sporozoites isolated from the salivary glands of infected An. stephensi on day 14 (c) post-infection with P. c. chabaudi AS (PCC AS) or P. c. chabaudi CB (PCC CB) ( $n=820$; data are presented as median with range). The number of sporozoites in the salivary glands of An. stephensi infected with PCC CB is significantly increased, as compared to those infected with Pcc AS ( $\mathrm{*} p=0.0222$ (MannWhitney test)). C57BL/ 6 mice with a gametocyte density of $\geq 0.1 \%$ of total erythrocytes were utilized on day 14 post-infection at a ratio of $\geq 1$ mouse to 100 mosquitoes. 

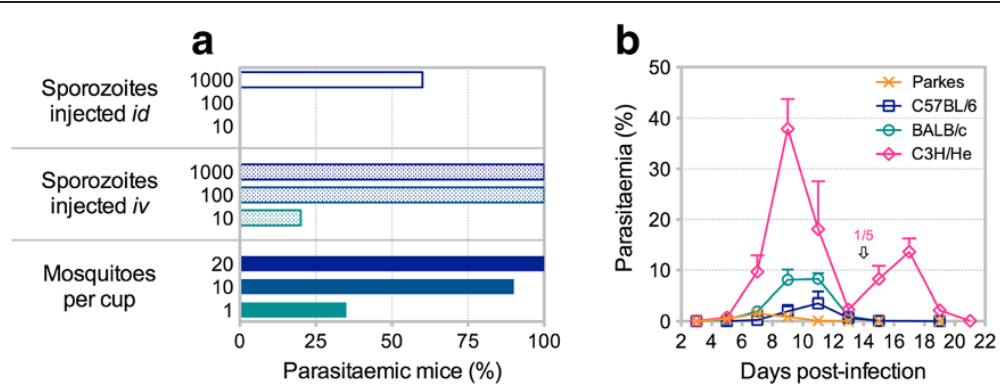

Figure 3 Transmissibility and virulence of Plasmodium chabaudi chabaudi AS in the mammalian host. a, Frequency of patent blood-stage parasitaemia in C57BL/6 mice injected id or iv with P. c. chabaudi AS sporozoites, or exposed to cups of P. c. chabaudi AS infected mosquitoes (n = 5-34 per group; pooled data from two independent experiments are shown). Parasitaemic mice were defined by thin blood smear, prepared daily between days 3 and 16 post-infection, with a limit of detection of $0.01 \% \mathrm{pE}$. b. Parasitaemia of Parkes, C57BL/6, BALB/c and C3H/He mice infected with P. c. chabaudi AS via mosquito bite ( $n$ = five per group; data are presented as mean with SEM). Peak parasitaemia is significantly increased in BALB/C mice, as compared to Parkes mice, and in C3H/He mice, as compared to BALB/C mice ( ${ }^{* *} p=0.0079$ (Mann-Whitney test)). Note that on day 14 post-infection one $\mathrm{C} 3 \mathrm{H} / \mathrm{He}$ mouse succumbed to infection.

patent blood-stage parasitaemia, whilst exposure to 20 mosquitoes ensures patent parasitaemia in $100 \%$ of mice (Figure 3a). Although the course of infection in mice infected via mosquito bite is similar to that observed in mice injected with sporozoites, the potential influence of the route of transmission on the pathogenesis of malaria is not yet fully understood. Mice are therefore routinely infected with $P$. c. chabaudi via the natural route, and exposed to $20 \mathrm{An}$. stephensi leading to an estimated 9.15 (6.9-13.6) (median with range) infective bites; of 117 wild-type C57BL/6 mice infected with P. c. chabaudi AS via mosquito bite, 116 mice (99.1\%) developed patent blood-stage parasitaemia. The pre-patent period in C57BL/6 mice infected with $P$. c. chabaudi AS via mosquito bite is $1(<1-6)$ (median with range) day(s) following liver merozoite egress; 39 of 45 mice (86.7\%) have a pre-patent period of $\leq 2$ days. To observe a pre-patent period of $\leq 2$ days in C57BL/6 mice infected with $P$. c. chabaudi AS via direct blood challenge would require iv injection of $\geq 10^{5} \mathrm{pE}$, approximating the number of liver merozoites that initiate the erythrocytic cycle following mosquito transmission. The resulting course of infection, and associated morbidity and mortality, within the mammalian host is strain dependent (Figure 3b); similarly, the course and outcome of infection is dependent upon the isolate of P. c. chabaudi inoculated via mosquito bite [Spence PJ et al. personal communication.]. Mosquito transmission of $P$. c. chabaudi therefore permits analysis of parasite virulence and host susceptibility to malaria, in the context of the complete Plasmodium life cycle and with infections initiated via the natural route.

\section{Discussion and conclusions}

Mosquito transmission of $P$. c. chabaudi according to this optimized protocol is highly efficient and reproducible, and facilitates routine transmission of distinct isolates of P. c. chabaudi with a success rate of $100 \%$. Optimal transmission is attained when An. stephensi feed on mice after the peak of asexual blood-stage parasitaemia, when circulating gametocyte densities surpass $0.1 \%$ of total erythrocytes. Thereafter, it is critical that infected mosquitoes are continuously maintained at $26.0^{\circ} \mathrm{C}$; unfed mosquitoes are therefore not removed from cages, and mosquito handling is minimized at all times. This inevitably leads to an apparent limitation of the protocol; the number of infected mosquito bites is not known for individual mice. Nevertheless, the process of sporozoite inoculation in the mammalian dermis during mosquito probing is stochastic $[9,10]$; the sporozoite inoculum in all individuals, including those exposed to a defined number of infected mosquito bites, will always be unknown. Importantly, the inoculum size is unlikely to be associated with disease severity or outcome [1113]. Indeed, it was noted in this study that the magnitude of peak parasitaemia is not influenced by the number of injected sporozoites, or the number of mosquito bites, that initiate infection. Thus, whilst in exceptional experimental circumstances it is necessary to control the pathogen dose via mechanical transmission of sporozoites [14], the majority of experimental studies of malaria should be initiated via mosquito bite, the natural route of sporozoite inoculation. In this context, it is of considerable importance that mosquito vector densities of $P$. c. chabaudi, unlike other rodent malaria species, are within the accepted physiological range associated with human Plasmodium infections of their natural vectors [15]. Mosquito transmission of $P$. c. chabaudi therefore provides an unparalleled opportunity to study both the mosquito vector and mammalian host stages of malaria within a single relevant infection setting. This protocol has been utilized to demonstrate that the vector directly regulates Plasmodium virulence during the erythrocytic cycle [Spence PJ et al. personal communication], and the 
consequence of vector regulation can clearly be observed when comparing the course of an infection initiated via mosquito bite, as opposed to following serial blood passage (Figure 2a and Figure 3b). It is therefore critical to perform experimental malaria studies, including those focused only on the erythrocytic cycle, in the context of the complete Plasmodium life cycle. Thus, mosquito transmission of Plasmodium should no longer be the exception but, rather, the rule in the field of experimental malaria.

\section{Competing interests}

The authors declare that they have no competing interests.

\section{Authors' contributions}

PJS, WJ and JL designed the study; PJS and WJ developed the protocol; PJS, WJ, PL and WN performed the experiments; PJS analysed the data; PJS wrote the manuscript; all authors read and approved the final manuscript.

\section{Acknowledgements}

This work was supported by the Medical Research Council (U117584248) and the Wellcome Trust (048684). PJS is the recipient of a Leverhulme Trust early career fellowship. WN is the recipient of an EVIMalaR PhD studentship. The authors thank R Sinden, K Baker and M Tunnicliff for provision of Anopheles stephensi and, together with J Thompson, for helpful discussions. The authors also thank I Tumwine and T Brugat for careful reading of the manuscript.

Received: 17 October 2012 Accepted: 3 December 2012

Published: 6 December 2012

\section{References}

1. Stephens R, Culleton RL, Lamb TJ: The contribution of Plasmodium chabaudi to our understanding of malaria. Trends Parasitol 2012, 28:73-82.

2. Mackinnon MJ, Bell A, Read AF: The effects of mosquito transmission and population bottlenecking on virulence, multiplication rate and rosetting in rodent malaria. Int J Parasitol 2005, 35:145-153.

3. Spence PJ, Cunningham D, Jarra W, Lawton J, Langhorne J, Thompson J: Transformation of the rodent malaria parasite Plasmodium chabaudi. Nat Protoc 2011, 6:553-561.

4. Mackinnon MJ, Read AF: Immunity promotes virulence evolution in a malaria model. PLOS Biol 2004, 2:e230

5. Ebert D: Experimental evolution of parasites. Science 1998, 282:1432-1435.

6. Landau I, Boulard Y: Life cycles and morphology. In Rodent Malaria. Edited by Killick-Kendrick R, Peters W. (London) Ltd: Academic Press Inc; 1978:53-84.

7. Fonseca $\mathrm{L}$, Seixas $\mathrm{E}$, Butcher $\mathrm{G}$, Langhorne J: Cytokine responses of $C D 4^{+} \mathrm{T}$ cells during a Plasmodium chabaudi chabaudi (ER) blood-stage infection in mice initiated by the natural route of infection. Malar J 2007, 6:77.

8. Malaria Research and Reference Reagent Resource Center. www.mr4.org.

9. Beier JC, Davis JR, Vaughan JA, Noden BH, Beier MS: Quantitation of Plasmodium falciparum sporozoites transmitted in vitro by experimentally infected Anopheles gambiae and Anopheles stephensi. AmJTrop Med Hyg 1991, 44:564-570.

10. Ponnudurai $\mathrm{T}$, Lensen $\mathrm{AH}$, van Gemert GJ, Bolmer MG, Meuwissen JH: Feeding behaviour and sporozoite ejection by infected Anopheles stephensi. Trans R Soc Trop Med Hyg 1991, 85:175-180.

11. Glynn JR, Bradley DJ: Inoculum size, incubation period and severity of malaria. Analysis of data from malaria therapy records. Parasitology 1995, 110:7-19.

12. Glynn JR, Collins WE, Jeffery GM, Bradley DJ: Infecting dose and severity of falciparum malaria. Trans R Soc Trop Med Hyg 1995, 89:281-283.

13. Jarra W, Hills LA, March JC, Brown KN: Protective immunity to malaria. Studies with cloned lines of Plasmodium chabaudi chabaudi and $P$. berghei in CBA/Ca mice. II. The effectiveness and inter- or intra-species specificity of the passive transfer of immunity with serum. Parasite Immunol 1986, 8:239-254.

14. Epstein JE, Tewari K, Lyke KE, Sim BK, Billingsley PF, Laurens MB, Gunasekera A, Chakravarty S, James ER, Sedegah M, Richman A, Velmurugan S, Reyes S, Li M, Tucker K, Ahumada A, Ruben AJ, Li T, Stafford R, Eappen AG, Tamminga C, Bennett JW, Ockenhouse CF, Murphy JR, Komisar J, Thomas N,
Loyevsky M, Birkett A, Plowe CV, Loucq C, Edelman R, Richie TL, Seder RA, Hoffman SL: Live attenuated malaria vaccine designed to protect through hepatic CD8 ${ }^{+}$T cell immunity. Science 2011, 334:475-480.

15. Sinden RE, Dawes EJ, Alavi Y, Waldock J, Finney O, Mendoza J, Butcher GA, Andrews L, Hill AV, Gilbert SC, Basanez MG: Progression of Plasmodium berghei through Anopheles stephensi is density-dependent. PLoS Pathog 2007, 3:e195.

doi:10.1186/1475-2875-11-407

Cite this article as: Spence et al:: Mosquito transmission of the rodent malaria parasite Plasmodium chabaudi. Malaria Journal 2012 11:407.

\section{Submit your next manuscript to BioMed Central and take full advantage of:}

- Convenient online submission

- Thorough peer review

- No space constraints or color figure charges

- Immediate publication on acceptance

- Inclusion in PubMed, CAS, Scopus and Google Scholar

- Research which is freely available for redistribution 